Document downloaded from:

http://hdl.handle.net/10251/95439

This paper must be cited as:

Martorell-Tejedor, S.; Palanca, S.; Angel Maquieira Catala; Tortajada-Genaro, LA. (2018). Blocked recombinase polymerase amplification for mutation analysis of PIK3CA gene. Analytical Biochemistry. 544:49-56. doi:10.1016/j.ab.2017.12.013

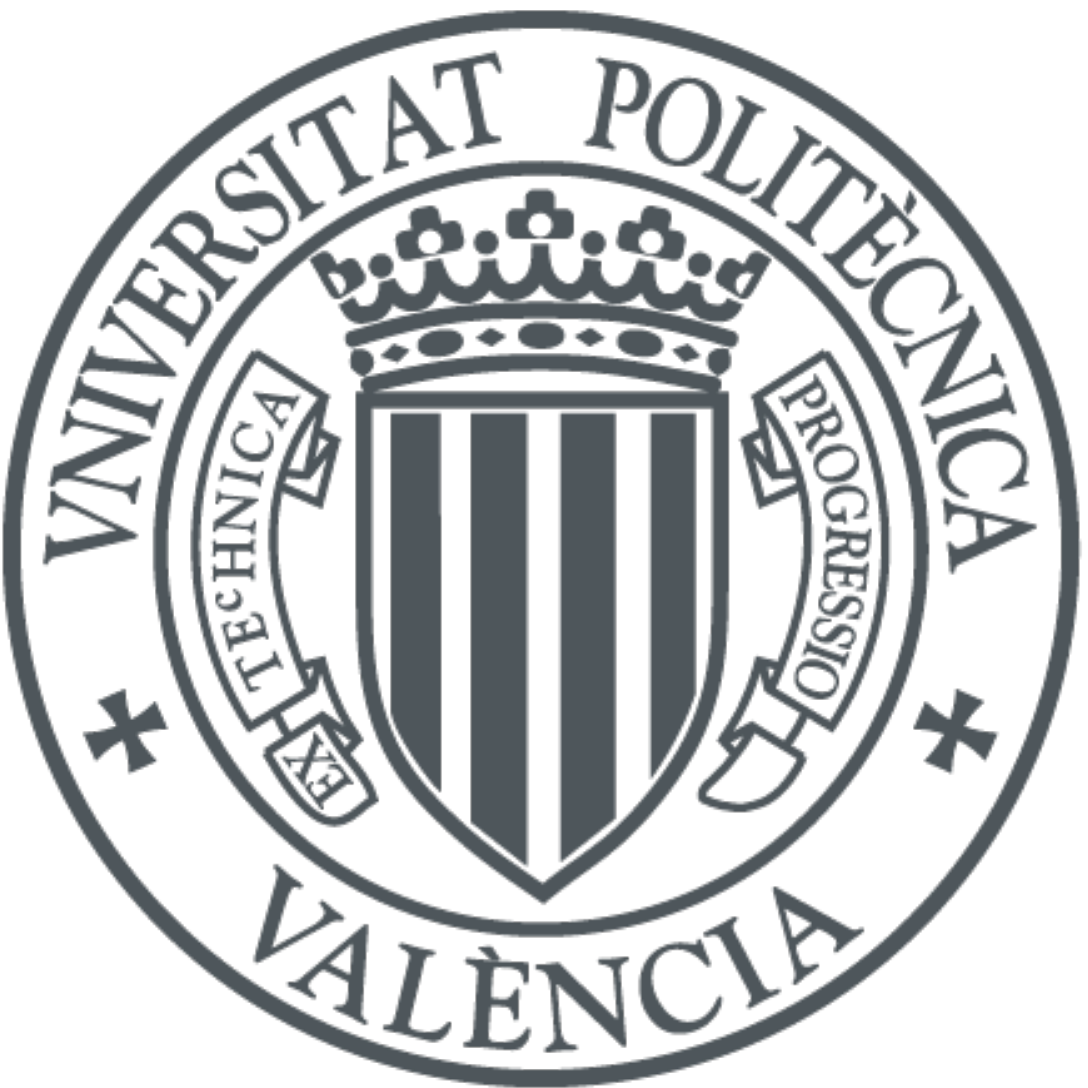

The final publication is available at

https://doi.org/10.1016/j.ab.2017.12.013

Copyright Elsevier

Additional Information 
3

4 Sara Martorell(a), Sarai Palanca ${ }^{(b)}$, Ángel Maquieira ${ }^{(a, c, d)}$, Luis A. Tortajada-Genaro ${ }^{(a, c, d)}$ 5

6

7

8

9

10

11

12

13

14

\section{Blocked recombinase polymerase amplification for mutation analysis of PIK3CA gene}

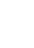

(a) Instituto Interuniversitario de Investigación de Reconocimiento Molecular y Desarrollo

Tecnológico (IDM), Universitat Politècnica de València-Universidad de València

(b) Servicio de Análisis Clínicos. Hospital Universitario y Politécnico La Fe. Av. Fernando

Abril Martorell, no 106, E46026, Valencia, Spain

(c) Departamento de Química, Universitat Politècnica de València, Camino de Vera s/n, 1 E46022 Valencia, Spain

2 (d) Unidad Mixta UPV-La Fe, Nanomedicine and Sensors, IIS La Fe, Valencia, Spain

3 Email: luitorge@qim.upv.es 


\section{ABSTRACT}

16 A blocked recombinase polymerase amplification (blocked-RPA) approach has been developed for the enrichment of mutated templates in heterogeneous specimens as tumor tissues. This isothermal amplification technique opens alternative solutions for meeting the technological demand of physician office laboratories. Herein, the detection of mutations in PIK3CA gene, such as p.E545K, and p.H1047L, is presented. The main element was an oligonucleotide (dideoxycytidine functionalized at 3'-end) which matched with wild-type sequence in the target locus. The amplification was performed operating at $37^{\circ} \mathrm{C}$ during $40 \mathrm{~min}$. The results demonstrated that the competition between the upstream primer and the blocker reduced the percentage of amplified wild-type allele, making the detection of the present mutation easier. For mutation discrimination, a fast hybridization assay was performed in microarray

27 format on plastic chip and colorimetric detection. This approach enabled the reliable 28 discrimination of specific mutations against a background of up to $95 \%$ wild-type DNA. 29 The applicability of the method, based on the combination of blocked-RPA and low-cost 30 chip hybridization, was successfully proven for the genotyping of various cancer cell lines as well as tumor tissues. The assignations agreed with those provided by nextgeneration sequencing. Therefore, these investigations would support a personalized approach to patient care based on the molecular signature of human cancers.

Keywords: recombinase polymerase amplification; blocking agent; mutations in PIK3CA oncogene; gel electrophoresis; colorimetric array 


\section{INTRODUCTION}

39 Neoplastic diseases are currently the leading cause of morbidity and mortality in developed countries, making cancer a public health problem of the first magnitude. The success of anticancer therapies depends on the correct assignation of disease subtype. Therefore, the detection of tumour biomarkers is important for the application of a personalized medicine [1,2]. Somatic mutations on oncogenes are excellent predictive biomarkers since the response to a particular line of treatment can be anticipated, reducing the adverse effects and improving efficiency [3]. They also can be prognostic biomarkers enabling the prediction of disease progression.

Several molecular techniques can be applied for knowing the mutational status of oncogene hotspots. In a DNA extract from patient sample (solid tissues and body fluids), the mutant variant is found in a low proportion compared to wild-type variant. This scenario presents an analytical challenge because wild-type variant can exhaust essential reagents and/or mask the mutant signal during detection assays [4]. Nextgeneration sequencing instruments holds great promise for point mutation detection, but currently, this technology is available in few health centers and clinics. The alternative solutions are based on introducing an enrichment method combined to more simple detection techniques. Particularly interesting are PCR-based methods proposed for a selective (or quasi-selective) amplification of minority alleles and mutations. These include the use of allele-specific primers to selectively initiate the amplification of the mutated genotype $[5,6]$; addition of oligonucleotide clamps to preferentially inhibit primer extension on wild type targets [7-9]; and control of temperature thermocycling to favor the preferential denaturation of mutant targets [10]. The differential behavior between wild-type and mutant variants are obtained using real-time or end-point amplification, fluorescence being the main detection principle. Most of described methods are able to detect the presence of mutation in the selected hotspot, but they do not provide information about their identification. Herein, we developed an in vitro method based on a blocked isothermal amplification as enrichment technique for analyzing point mutations in PIK3CA gene (phosphatidylinositol-4,5-bisphosphate 3-kinase, catalytic subunit alpha) as oncogene model. This gene codifies important cell membrane element and second messenger involved in cell signaling. The mutation has been located in human cancer as colorectal, breast, glioblastoma, gastric, ovary, lung, and skin [11,12]. There is an important basic 
survival, motility, and metabolism [13]. Additionally, novel inhibitor drugs are targeting this mutant protein [14].

Isothermal amplifications are revolutionizing the development of point-of-care testing due to their capability for the integration in portable, inexpensive instruments or devices $[15,16]$. Loop-mediated isothermal amplification (LAMP) is the most cited alternative to PCR. Their allele-specific option has displayed excellent results in the detection of a specific point mutation in EGFR gene [17], BRAFgene [18] and KRAS gene [19]. Rolling circle amplification (RCA) and recombinase polymerase amplification (RPA) have been used for the detection of point mutation [20, 21]. In our previous research, the genotyping of a single-point polymorphism was achieved using allele-specific primers for discriminating perfect-match and mismatch allele. In the current study, a new strategy is addressed for improving sensitivity, based on the addition of a blocking oligonucleotide to reduce the amplification of wild-type variant. Then, the presence of mutated variants can be established. Furthermore, an allele-selective hybridization is proposed in order to discriminate between different nucleotide changes. As proof of concept, the selected format involves a plastic chip as analytical platform and colorimetric imagining as detection approach. Microarrays represent an accurate tool for parallel identification of multiple markers, suitable for routine analysis in medical diagnostics [22].

\section{EXPERIMENTAL}

\section{Material and reagents}

Oligonucleotide sets were designed for the analysis of two important hotspots in PIK3CA gene (Table SI.1). In the helicase domain of exon 9, the most frequent mutations are in codon 542 (p.E542K) and codon 545 (p.E545K, p.E545A, and p.E545G). In the kinase domain of exon 20, they are in codon 1047 (p.H1047R and p.H1047L). Thermodynamic parameters were calculated for inducing the selective recognition of target regions [23]. The list of used oligonucleotides, supplied by Eurofins, is included as Supplementary Material (Table SI.2).

100 The reagents used for genomic DNA amplification were TwistAmp Basic RPA kit 101 (TwistDx, UK). For microarray detection, the printing buffer composition was (2-(Nmorpholino)ethanesulfonic acid at $0.1 \mathrm{M}$, 1-ethyl-3-(3-dimethylaminopropyl) carbodiimide at $20 \mathrm{mM}$, glycerol $10 \%$ (pH 5.5). The hybridization buffer was salinesodium citrate (SSC) 2x: sodium chloride at $150 \mathrm{mM}$, sodium citrate at $15 \mathrm{mM}$, 
$106 \mathrm{~L}^{-1}$, trisodium citrate $1.5 \mathrm{mmol} \mathrm{L}^{-1}$. Developing buffer was a phosphate buffered saline 107 solution (PBS-T) containing $137 \mathrm{mM} \mathrm{NaCl}, 12 \mathrm{mM}$ phosphate, $2.7 \mathrm{mM} \mathrm{KCl}, 0.05 \%$ 108 Tween $20(\mathrm{pH} 7.4)$.

109 The hybridization chips were prepared by immobilizing the allele-specific probes on 110 rectangular slides $(25 \mathrm{~mm} \times 75 \mathrm{~mm})$. For covalent anchoring of probes, polycarbonate 111 surface was activated by UV-ozone incubation (model FHR.Clean.150-Lab, FHR, 112 Germany) using UV lamps $\left(50 \mathrm{~mW} \mathrm{~cm}^{-1}\right)$ at $254 \mathrm{~nm}$. After, the chips were immersed in 113 a solution of $\mathrm{NaOH} 1 \mathrm{M}$ at $60^{\circ} \mathrm{C}$, washed with distilled water and dried. The dispensation 114 of amine-DNA probes in printing buffer on the modified surface was performed by non115 contact array printer equipment (AD1500, Biodot). The printing volume drop was $40 \mathrm{~nL}$. 116 Six arrays were printed per chip, including 4 replicates per target probe and controls. The 117 chips were incubated for 1 hour, washed with PBS-T and water, dried and stored at $4{ }^{\circ} \mathrm{C}$ 118 until use.

\section{Samples and DNA extraction}

Patients and several volunteers were recruited for the present study according to ethics and with informed consents. Genomic DNA was extracted from three 5- $\mu$ m thick FFPE sections using Deparaffinization Solution and the GeneRead DNA FFPE Kit (Qiagen, Hilden, Germany) according to manufacturer's protocol. This isolation kit contains uracilDNA glycosylase (UDG) that leads to the reduction of $C>T$ sequence artefacts. Buccal cells were collected by rolling the swab (Catch-All sample collection swab, Epicenter) on the inside of the cheek. DNA extraction was performed using the PureLink Genomic DNA Mini Kit (Invitrogen). Briefly, the swab was incubated with Proteinase K, RNAse and lysate buffer. The purification was performed by using a spin column-based centrifugation procedure. Human cell lines SK-N-AS (ATCC CRL-2137) and HCT 116 (ATCC CCL-247) were used as native (wild type) and heterozygous (p.H1047R/c.3140A>G) controls, respectively. In these cases, DNA extraction was performed using PureLink kits (Invitrogen).

134 In addition, the assay included a negative control (DNA from Salmonella Typhimurium). 135 The concentration of the DNA extracts $\left(n g \mu \mathrm{L}^{-1}\right.$ ) was obtained by spectrophotometry 136 using NanoDrop 2000c, and by fluorimetry using Qubit dsDNA HS Assay Kit 137 (ThermoFisher Scientific). 
PCR amplification in single format was performed for each target hotspot in PIK3CA gene (exon 9 or exon 20). Each reaction mixture contained $1 \times$ DNA polymerase buffer,

$1423 \mathrm{mM}$ of $\mathrm{MgCl}_{2}, 200 \mu \mathrm{M}$ of each deoxynucleotide triphosphate, $200 \mathrm{nM}$ of upstream 143 primer and downstream digoxigenin-labeled primer, $4 \mathrm{ng}$ of genomic DNA, and 1 unit of 144 DNA polymerase (Biotools, Madrid, Spain). In certain experiments, a blocking 145 oligonucleotide complementary to wild-type variant was added to each mixture (10 nM $146400 \mathrm{nM}$ ). The reaction was performed in thermocycler (United Nations, VWR) under the 147 following conditions: initial denaturation cycle of $95{ }^{\circ} \mathrm{C}$ for $5 \mathrm{~min}, 35$ cycles of 148 denaturation at $95^{\circ} \mathrm{C}$ for $30 \mathrm{~s}$, annealing $57^{\circ} \mathrm{C}$ for $30 \mathrm{~s}$ and elongation at $72{ }^{\circ} \mathrm{C}$ for $60 \mathrm{~s}$, 149 finally one cycle extension at the end for $5 \mathrm{~min}$.

\section{RPA amplification}

RPA amplification in single format was performed for each target hotspot in PIK3CA gene (exon 9 or exon 20 ). The reaction mixtures $(12.5 \mu \mathrm{L})$ were prepared with rehydrated buffer, $14 \mathrm{mM}$ of magnesium acetate, $480 \mathrm{nM}$ of upstream primer and downstream digoxigenin-labeled primer, $50 \mathrm{nM}$ of blocking agent, $4 \mathrm{ng}$ of genomic DNA, and the enzyme pellet. The reaction mixtures for conventional RPA did not contained the oligonucleotide complementary to wild-type variant. The heating system used was a thermocycler (United Nations, VWR) at $37^{\circ} \mathrm{C}$ for $40 \mathrm{~min}$.

\section{Gel electrophoretic detection}

The RPA amplicons were cleaned for being visualized using agarose gel electrophoresis. Two methods were compared, the first being based on silica-gel membrane adsorption (PCR purification kit, Jena Bioscience, Germany). The RPA products, mixed with binding buffer and isopropanol, was transferred to the activated column and centrifuged at $10.000 \mathrm{~g}$ for $30 \mathrm{~s}$. After washing the column twice, the elution fraction was collected in distilled water. The second method was based on denaturation protocol. The RPA products were incubated at $65^{\circ} \mathrm{C}$ in dry bath (model FB 15103, Fisher Scientific) for 10 min. After centrifugation at $10.000 \mathrm{~g}$ for $20 \mathrm{~s}$, the supernatant fraction was collected.

The electrophoretic separation was conducted in agarose gel $3 \%$. Amplification solutions were mixed with loading buffer and transferred to gel wells. After separation at 110 V, fluorescent dye (Realsafe Nucleic acid Staining Solution 2x, Real Lab.) was incubated for $30 \mathrm{~min}$. Gel images were captured using a smartphone Iphone 7 and analyzed using ImageJ software. 


\section{Colorimetric chip detection}

176 The RPA products were detected and identified by a hybridization assay using allele 177 selective probes immobilized on polycarbonate chips. Amplified products $(5 \mu \mathrm{L})$ were 178 mixed with $45 \mu \mathrm{L}$ of hybridization buffer, heated $\left(95^{\circ} \mathrm{C}, 5 \mathrm{~min}\right.$ ) and dispensed onto 179 sensing arrays to perform the simultaneous analysis of 6 samples (four probe replicates). 180 After incubation $\left(37^{\circ} \mathrm{C}, 60 \mathrm{~min}\right)$, the arrays were rinsed with progressive dilutions of 181 hybridization washing buffer. To develop the probe-product duplex, an immunoreaction 182 was used. PBST solution with 1:2500 monoclonal anti-Dig antibody (Abcam) and 1:400 183 monoclonal antisheep-HRP antibody in PBST, was dispensed (room temperature, 30 184 min). As HRP-substrate, 3,3',5,5'-tetramethylbenzidine solution (ep(HS)TMB-mA, SDT 185 Reagents) was selected, generating a solid deposit.

186 Chips were directly scanned (Epson Perfection 1640SU office scanner), producing gray187 scale images (Tagged Image File Format, color depth 16 bit, scale 0-65535). The optical 188 intensity signals of each spot were quantified using in-home software. Image processing 189 (feature gridding, addressing, segmentation and quality assurance) was automatically 190 performed in less than $5 \mathrm{~min}$.

\section{Reference method}

193 Several methods for detecting somatic mutations are currently available. NGS, 194 considered to be an optimum method for mutation detection, was conducted employing 195 the lon Torrent PGM technology (ThermoFisher Scientific). The Oncomine Solid Tumor 196 DNA kit was used for DNA analysis (ThermoFisher Scientific). This assay simultaneously 197 screens hotspots mutations in 22 genes (included PIK3CA). For generating the DNA 198 barcoded libraries, a multiplex PCR amplification of $10 \mathrm{ng}$ of genomic DNA was 199 performed. Sequencing was performed on the Ion Torrent PGM system on a 318v2 Ion 200 Chip using Ion PGM Sequencing Hi-Q kit (Thermo Fisher Scientific). Data from 201 sequencing runs were transferred to the Torrent Server, alignment to the hg19 human 202 reference genome and variant calling was performed by the lon Torrent Suite Software 2035.4 (Thermo Fisher Scientific). In addition, all identified variants, such as PIK3CA 204 mutations in exon 9 and 20 (p.E545K and p.H1047L), were visually checked using the 205 Integrative Genomics Viewer (IGV) software. 


\section{RESULTS}

208 Assay design

209 A flow diagram showing the main steps in our assay for the detection of point mutations 210 is illustrated in Figure 1. From total genomic DNA extracted from human tissues, the 211 target sequence is amplified following a clamp blocked RPA reaction. Compared to 212 conventional RPA method, the reaction solution also contained an oligonucleotide 213 complementary to wild-type DNA (blocker), the target nucleotide being in the central 214 position of oligonucleotide. The upstream primer is in the vicinity of blocking 215 oligonucleotide, and partially overlaps its 5'-end. The blocker oligonucleotide is designed 216 for producing more stable complexes than upstream primer. The method operates by a 217 competition for a common target site.

219 Figure 1. Schematic illustration of the RPA reaction for minority allele enrichment based 220 on the addition of blocking agent. (Up) Wild-type blocked amplification. (Bottom) Mutant 221 amplification.

222

223 The amplification mechanism of mutant variants is based on the action of recombinases ( $\mathrm{T} 4 \mathrm{uvs} \mathrm{X}$ and loading factor T4 uvs Y), which form complexes with primers and bind them with their homologous sequences in duplex DNA [24]. A single-stranded DNA binding (SSB) protein binds to the displaced DNA strand and stabilizes the resulting D loop. Bsu polymerase (large fragment of Bacillus subtilis Pol1) produces the primer extension from the 3'-end, replicating mutant allele. The amplification of wild-type variants is impaired due to the presence of the blocking oligonucleotide. In this case, the blocking oligonucleotide dominates the binding to the template over the primer. Since the blocker also contains a chemical modification at the 3'-end, the oligonucleotide cannot be extended by Bsu polymerase. Then, the addition of this blocking agent during amplification of the target region induces the competition for the binding site in DNA template, leading to a preferential amplification of minority mutations. This clamping effect has been previously described for PCR-based methods [12,13], but this is the first time for RPA. 


\section{RPA amplification}

240 The proposed assay was tested for the genotyping of the main hotspots in PIK3CA gene

241 (codon 542/545 and codon 1047). An in-silico design was applied for the selection of the

242 sequence-specific primers based on the requirements established for the product 243 properties and method performances. Compared to the conventional RPA methods, 244 short primers ( $<30$ nucleotides) were selected to facilitate the subsequent allele-specific 245 assay. Even though they had little recombinase-mediated strand-invasion activity, short 246 primers can still function via hybridization recognition [25]. An additional design 247 restriction was taken into account. The length of products was limited to short 248 amplification products (lower than $100 \mathrm{bp}$ ). As the conservation of biopsied tissues as 249 formalin-fixed and paraffin-embedded can lead to an important degradation of nucleic 250 acids, this selection criteria can minimize false-negative results.

251 Using the selected oligonucleotide sets (upstream and downstream primers), the first 252 experiments were focused on the optimization of reaction conditions for the amplification 253 of both hotpots in single format. Regarding kinetic behavior, the amplification process 254 reached a stationary phase after $40 \mathrm{~min}$. A similar product formation was obtained when 255 the RPA reaction was produced within the $37-42{ }^{\circ} \mathrm{C}$ range, showing high tolerance to 256 temperature fluctuations. Therefore, the selected conditions were similar to previous 257 studies for human cancer tissues [26].

258 RPA specificity was checked on the basis on the detection of target regions from human 259 and non-human genomic DNA. Negative results were reported for the non-target genes 260 or samples, demonstrating the absence of false-positive results. The matrix effect was 261 also examined, analyzing different samples (cell culture, buccal swap, colon tissue) and 262 storage conditions (fresh tissues and formaldehyde fixed-paraffin embedded tissues). 263 Under the selected extraction and amplification conditions, excellent end-point 264 amplification yields were obtained. A detectable signal was measured for $5 \cdot 10^{2}$ DNA 265 copies. The results were comparable to those obtained with the PCR-based method, 266 independently on sample source (paired t-test, $p$-value $<0.05$ ).

\section{Selection of blocking oligonucleotides}

269 A preferential RPA-based amplification of minority mutations was studied based on the 270 addition of a blocking oligonucleotide to the reaction solution. Following the modalities 271 described for blocked PCR amplification, two kinds of oligonucleotide sets were assayed. 272 The first approach, both oligonucleotides (upstream primer and blocking agent) are 
273 complementary to different template regions, preventing the amplification in the 274 elongation step. In the second approach, also called clamp strategy, the blocker partially 275 overlaps with the upstream primer, reducing the amplification in the primer annealing 276 step.

277 As the number of possible oligonucleotides can achieve hundreds, the thermodynamic 278 stability of DNA duplexes was examined. The parameters were the variation of free 279 energy associated to the formation of DNA duplexes $(\Delta G)$ and melting temperature $\left(T_{m}\right)$, 280 or temperature at which half of the blocking oligonucleotides are single-stranded state $281(0.1 \mathrm{M} \mathrm{NaCl}, 25 \stackrel{\circ}{\circ} \mathrm{C}$ at $\mathrm{pH}=7)$. Firstly, the formation of upstream primer duplexes was 282 studied in both hotspots of PIK3CA gene. The $\triangle \mathrm{G}$-values for the selected primers ranged 283 between $-19.8 \mathrm{kcal} \mathrm{mol}^{-1}$ and $-20.5 \mathrm{kcal} \mathrm{mol}^{-1}$, corresponding to $\mathrm{T}_{\mathrm{m}}=58.6-60.9 \stackrel{\circ}{ }{ }^{\circ} \mathrm{C}$. 284 Secondly, the effect of design parameters on the blocking oligonucleotide/template 285 hybrids was estimated. Blocking agents with length higher than 22 nucleotides produced 286 DNA complexes more stable than the selected primers $\left(T_{m}>62^{\circ} \mathrm{C}\right)$. The differential 287 variation of free energy associated to the formation of DNA duplexes (wild-type vs. 288 mutant variants) was calculated in function of clamp-region length and mutation position. 289 The nucleotide number that overlapped with the primer produced a slight effect on the 290 discrimination recognition for perfect match complex (wild-type template) respect to the 291 mismatched complexes (mutant templates). However, the position of mutation on 292 blocking agent sequence varied drastically the stability of the mismatched hybrids. In 293 fact, the maximum difference between blockers and wild-type/mutant complexes was 294 achieved when the mutations was located in a central position.

295 On the basis on these experiments, several design requirements of blocking 296 oligonucleotide were defined for blocked RPA assay (Table 1). Applying in silico 297 calculations, two primers and a blocking oligonucleotide compose the selected set per 298 studied mutation. The estimated $\Delta$ G-values for wild-type complex were $-23.6 \mathrm{kcal} \mathrm{mol}^{-1}$ 299 for exon 9 and $-21.9 \mathrm{kcal} \mathrm{mol}^{-1}$ for exon 20 , corresponding to $T_{m}=64.5^{\circ} \mathrm{C}$ and $\mathrm{T}_{\mathrm{m}}=63.3$ $300 \stackrel{\circ}{\circ}$, respectively. The selected blocking oligonucleotides should form a more stable 301 complex with wild-type than with the mutated variant (about $4 \mathrm{kcal} \mathrm{mol}^{-1}$ ). Regarding the clamp effect, the common nucleotides (3'-end of primer and 5'-end of blocking agent) were 2 in both targeted hotspots. Under these conditions, the expected reduction of 305 Although RPA mechanism is based on the action of several enzymes, a differential 306 behavior was expected. The blocker would preferentially hybridize onto the wild type 307 template strand and the upstream primer would bind to the mutant template. 
Table 1: (a) Design criteria of blocking oligonucleotide. (b) Optimized variables for blocked RPA.

\section{Set-up of blocked amplification}

312 The following step was the study of experimental conditions for blocked RPA, using DNA 313 extracts from control/mutant cell lines and the selected olignucleotides. In a first set of 314 RPA experiments, reaction mixtures contained the downstream primer and the 315 unmodified blocker (without upstream primer). Positive responses were observed, 316 indicating that the blocking oligonucleotide acted as upstream primer (Figure 2a). Later, 317 the same RPA reactions were performed, including modifications at the 3 '-end of the 318 blocker (capped extension). The studied modifications were dideoxycytidine (ddC) and 319 non-sense 3-mer tail (not complementary to template molecule), leading a signal reduction (t-test: $p$-value $<0.002$ ). Both chain terminator avoided the 3 '-extension, yielding similar responses to negative controls, even for high concentrations of blockers (t-test: $p$-value>0.95). For further assays, ddC modified blocker was selected.

323 Human genomic DNA was added in PCR and RPA reactions containing three 324 oligonucleotides (primers and blocker) for non-clamp and clamp approaches (Figure 2b). 325 In case of PCR, the addition of the blocker reduced the amplification of wild-type variant, 326 the effect being higher for clamp approach. These results agreed with those previously described for PCR-based methods, where the overlapping region between primer and blocker provided better assay performances $[7,8]$. In case of isothermal method, using a primer complementary to a different region than blocker, the primer elongation of wildtype DNA was produced ( $t$-test: $p$-value $=0.89$ ). The amplification of wild-type DNA only was reduced for clamp option (t-test: $p$-value $=0.01$ ). This differential blocking effect compared to PCR agreed with the expected features of RPA mechanism. In the first approach, recombinase mediated in the formation of both complexes (primer/template and blocker/template). However, polymerase used in RPA (Bsu polymerase) has stranddisplacing activity that means the ability to displace downstream DNA encountered during synthesis. Although the blocking oligonucleotide was initially bound, the primer elongation was possible, replicating wild-type templates. In case of second strategy, the observed behavior fitted with a physico-chemical competition for the same template region. The blocker operates a competitor of the upstream primer for the common target site. As the stability of blocker was higher, the primer annealing in RPA process was interfered. As the non-clamp option was incompatible with RPA biochemistry, further experiments were performed using the oligonucleotide set based on clamp effect. 
344 Figure 2: (a) Modification of blocker oligonucleotide for the prevention of Bsu extension 345 in a RPA mixture without upstream primer. Blocker concentration: 300, 600 and $800 \mathrm{nM}$.

346 (b) Amplifications (PCR and RPA) performed in conventional and blocked formats 347 (blocker concentration 200 nM). T-test: * $p$-value $<0.05,{ }^{* *} p$-value<0.01, *** $p$ value<0.001. Wild-type genomic DNA: 1300 copies.

Agarose gel electrophoresis was used for confirming the blocking effect on PCR and RPA reaction for PIK3CA (Figure SI.1). In case of PCR, the expected bands were detected (83 pb for exon 9 and 79 pb for exon 20). However, RPA products yielded smear bands due to mixture components (i.e. DNA binding proteins, detergents). Therefore, two post-amplification treatments were assayed (column purification and protein denaturation). The first method involved that silica-membrane columns retained short DNA molecules under high-salt conditions and eluted them using a low-salt buffer. The second method consisted in the denaturation of proteins at high temperature. Although both treatments provided a single band located at the estimated position, the separation obtained after heating strategy led to band intensities comparable to the PCR results. Further experiments showed that the band intensity decreased in the presence of blocking nucleotide in RPA reactions, being undetectable for high concentrations. Finally, the kinetic profile in the presence of blocker was compared to the conventional profile. Although amplification yield was lower than conventional value (about $50 \%$ ), the maximum signal was achieved after $40 \mathrm{~min}$ of reaction in both cases. Therefore, gel electrophoresis results proved the variation of replication activity as the consequence of blocker binding to the template.

367 For the selection of blocker concentration, genomic DNA extracted from human cell cultures (wild-type and mutant) were amplified, varying up to $400 \mathrm{nM}$ (stoichiometric ratio of 1.33 compared to the upstream primer). End-point responses decreased as the concentration of blocking oligonucleotide increased for both kind of templates (Figure 3). As the amplification variation was also observed in mutated variants, an unspecific interaction of blocker was produced in the mismatched templates. Nevertheless, certain reaction mixtures produced a nearly null signal for native variant and perfectly detectable signal for mutant variant. Experimental data fitted to a typical four-parameter logistic curve with a suitable model goodness $\left(R^{2}=0.94\right.$ and 0.98 , exon 9 and 20 , respectively).

376 The equation of this nonlinear regression was Signal $=d+(a-d) /\left(1+([b / o q] / c)^{b}\right)$, where 377 [blog] is the concentration of blocking agent, $d$ is the background signal, $a$ is the signal 
378 for the absence of blocking oligonucleotide, $c$ is related to the concentration at the 379 inflection point and $d$ is related to the curve steepness. Table 1 displays the estimated 380 values for both hotspots of PIK3CA gene. Significative differences were found in the 381 value of $c$-parameter that is related to half maximal effective concentration (EC50) or 382 blocking concentration that reduced the signal to half. The EC50 values for wild-type 383 templates were about 2 times lower than the values calculated for mutant templates for 384 both target regions. The observed differential behavior confirmed that the addition of 385 blocking agent produced a higher reduction of RPA reaction on perfect-match template 386 (native) than a mismatched template (mutant). A concentration of $50 \mathrm{nM}$ was selected 387 for further experiments.

388

389 Figure 3: Effect of blocking concentration on the end-point response of RPA-based 390 methods: Reactions for exon 9 (a) and exon 20 (b). Discontinue lines correspond to the 391 regression curve applying four-parameter logistic model.

392 Table 2: Regression parameters obtained applying four-parameter logistic model to 393 experimental data: blocking concentration vs. end-point response of RPA-based 394 methods.

395

\section{Identification of mutations}

397 The presence of a mutation was established from the results (electrophoresis or 398 fluorescence) for blocked RPA reactions (single format). Then, the detection of a band 399 indicated that a mutated variant was amplified, achieving a selectivity of $100 \%$.

400 The determination of the specific genetic variant needed an additional end-point method 401 (i.e. chip hybridization, bead hybridization, among others). In the present study, a simple 402 colorimetric microarray assay based on using allele specific probes was developed for 403 discriminating each specific mutation for a particular hotspot (Figure SI.2). The resulting 404 RPA amplicons were hybridized to the chip which carried probes complementary to the 405 targeted sequence. The use of microarray detection of RPA products has been 406 previously described in previous studies [21,27]. Nevertheless, the method was further 407 improved to serve the purpose of integrated mutational analysis.

408 Pursing the goal of a point-of-care test, a low-cost DNA chip combined with consumer 409 electronic device for the readout of results, was developed. Firstly, specific probes were 410 designed to maximize the selective recognition process, considering thermodynamic 
411 calculations (Table SI.3). The estimated free energy variation for the complexes between

412 the specific probes to its fully complementary target sequence varied from -21.4 to -22.4

$413 \mathrm{kcal} \mathrm{mol}^{-1}$ for exon 9 and from -21.7 to $-22.4 \mathrm{kcal} \mathrm{mol}^{-1}$ for exon 20 . The values calculated

414 for mismatched complexes ranged between -14.0 to $-20.0 \mathrm{kcal} \mathrm{mol}^{-1}$. Then, the estimated

415 differences were large enough for a discriminatory assay. Secondly, the probe

416 immobilization on polycarbonate chips was examined via photochemical surface

417 activation [28,29]. Under selected conditions, the conjugation of amine-functionalized

418 probes to carboxylate groups on chip surface produced an amide bond. The calculated

419 immobilization probe density was $3.5 \mathrm{pmol} \mathrm{cm}^{-2}$. A stable and strong bound was not

420 affected by $\mathrm{pH}$, temperature or microfluidic flows (losses $<5 \%$ ).

421 Latterly, the hybridization experiments were performed by incubating the RPA products

422 on chips with allele specific probes immobilized in microarray format. So, the

423 discriminating elements were spatially separated, but integrated in a single assay. The

424 optimization criteria were a high response signal for perfect-match probe and minimal

425 response for the mismatched probes. Therefore, the mutation can be identified due to

426 the formation of a perfect-match hybrid between the RPA product and one specific probe.

427 The composition of hybridization solution (formamide $20 \%$, low ionic strength) was

428 critical for the restrictive recognition. Another important factor was the volume of RPA

429 product dispensed on chip. A beneficial effect of assay performed in a microarray format

430 is the reduced distance that molecules need to travel from the bulk solution to the solid-

431 liquid interface. Selected volume $(45 \mu \mathrm{L})$ minimized the time required to obtain high spots

432 signal, associated to shortened diffusion times. Compared to PCR chip, higher

433 background signals were registered for RPA products. However, the signal-to-noise ratio

$434(\mathrm{~S} / \mathrm{N})$, calculated as the ratio of the signal and the standard deviation of the background 435 noise, was between 12 and 22.

436 Under the selected conditions, unblocked RPA products from human cell cultures were 437 hybridized on the chip. Given the presence of the biorecognition product, a variation of 438 the reflection properties of chip surface was measured (Figure 4). If there was no reaction 439 product, the maximum intensity of the reflected beam was collected (background signal). 440 If target gene-probe recognition and subsequent solid deposit formation occurred, the 441 light would strike the product, modifying the optical response. Wild-type products yielded 442 a high response for their specific probes $(S / N>15)$, and were low or null for the remaining 443 ones $(S / N<6)$. Mutant products of exon 9 (p.E545K) were specifically recognized for the 444 corresponding probe $(S / N=12)$, since the spot signals were significantly higher than 445 controls (t-test: $p$-value $=0,005)$. Mutant products of exon 20 ( $p . H 1074 R$ ) hybridized to 
446 two probes (wild-type and p.H1074R probe), giving detectable signals $(\mathrm{S} / \mathrm{N}>15)$ with 447 similar intensities (t-test: $p$-value $=0,07$ ). These values agreed with a genomic DNA 448 coming from a human cell culture that is heterozygote for this locus. Therefore, a 449 sensitive and selective hybridization assay was achieved. The results obtained using a 450 planar polycarbonate chip and a simple optical detection also demonstrated that the 451 assay is compatible with a typical mass produced material and a sensing technology of 452 DNA devices [16,22,30].

453 Figure 4: Probe Selectivity: spot intensity of array probes obtained from different 454 unblocked RPA reactions and samples. Dashed line indicates control negative response.

\section{Patient analysis}

There were important analytical challenges associated to the mutation detection and identification related to solid cancer screening. DNA from FFPET used to determine mutation status was highly degraded due to fixation. In fact, the nature of clinical sample and the conservation mode led to low amount/poor-quality of DNA in same samples. Intact double strand DNA ranged from 10 to $270 \mathrm{ng} \mu \mathrm{L}^{-1}$, with a purity $260 / 280 \mathrm{~nm}$ ranged from 1.7 to 2.1. In addition, the small proportion of mutated DNA can limit the success of the assay. So, assay sensitivity was determined by preparing heterozygous mixtures with increasing percentages of mutant DNA compared to the wild-type type (Figure SI.3). These experiments emulated the wide range of clinical scenarios regarding the variable proportion of tumor cells (mutant DNA) respect to non-tumor cells (native DNA) in a biopsied sample. Experimental data was adjusted to a linear regression with an excellent model goodness $\left(\mathrm{R}^{2}=0.994\right)$, indicating that blocked RPA produced a proportional amount of mutated sequences to the initial concentration of mutant genomic DNA. Mismatched DNA was detected up to $5 \%$, which indicated that the system was capable of detecting the mutant variant, even in low concentrations. In absence of blocker, the mutant variants were only detected when the percentage was 2-8 times higher. These results confirmed the formation of a stable duplex between blocker and wild-type

474 template, limiting the primer hybridization and consequently producing the mutant 475 enrichment.

476 Intra and inter-day reproducibility, expressed as the relative standard deviation of spot 477 intensities for the replicated assays (five replicates), were $13 \%$ and $17 \%$, respectively. 478 The ANOVA test showed that the end-point responses were comparable for the four 479 studied genes ( $p$-value $>0.05)$. 
The next experiments were focused on the analysis of blind samples collected from oncological patients (formalin fixed and paraffin embedded tissues). For each sample, a blocked RPA reaction per exon was performed. The arrangement of the microarray matrixes on the chip was designed in such a way that 6 samples (4 spot replicates) would be analyzed in parallel. The incorporation of quality controls (positive and negative) helped to ensure reliable results considering the possible variation of analytical process. Examples of the obtained microarray images are shown in Figure 5. Despite of the blocked amplification, positive responses for both wild-type probes were observed in all chips (S/N 6 \pm 2$)$. Nevertheless, a clear assignation was achieved based on the spot signals for mutant probes. Most of samples were assigned as wild-type for both studied locus (exon 9 and exon 20) because the mutant probe intensities were comparable to negative controls. The exceptions were two chips that showed a detectable response for one of the mutant probes. The $S / N$ were $10 \pm 2$ for p.E545K mutant and $17 \pm 2$ for p.H1047R mutant. Therefore, patients were classified as mutant in exon 9 and mutant in exon 20, respectively. The assignations agreed with those obtained using nextgeneration sequencing technology in all cases. However, sequencing analysis required higher amount of DNA, labor-intensive sample preparation and took longer to generate data compared with our method. The results highlighted that blocked RPA was an adequate approach since the copy number of mutated regions was high enough to be detected and discriminated in a hybridization assay. Furthermore, the proposed method fulfilled the requirements for a mutational analysis in a simple health system framework (i.e. short analysis time, low cost, and simple).

Figure 5: On-chip hybridization results for oncological patient samples: (a) Microarray layout, (b) Blocked RPA for exon 9, sample: wild-type, (c) Blocked RPA for exon 9, sample: mutant p.E545K, (d) Blocked RPA for exon 20, sample: wild-type, and (e) Blocked RPA for exon 20, sample: mutant p.H1074R.

\section{CONCLUSIONS}

Increasing knowledge on genetic variants and availability of specific therapeutic agents is enabling the development of a more personalized oncogenic medicine (e.g. specific monoclonal antibodies). However, a parallel technological development is required for translating them to clinic routine. Real-time PCR, droplet digital PCR and DNA sequencing are the most widely used method for mutational analysis. Although this objective has been effectively addressed, they involve a labor-intensive expensive 
515 solution or a limited capability in multiplex analysis. This study is aligned with the

516 research line of developing alternative solutions.

517 RPA-based methods are a successful approach for supporting portable diagnostic DNA 518 assays due to their performances. However, this study is the first in demonstrating that 519 blocked RPA is an adequate mutant enrichment technique. The results have shown that 520 this blocking behavior is a less effective process compared to other approaches, but the 521 achieved sensitivity (about $5 \%$ ) and reproducibility (about $15 \%$ ) are enough for their 522 application to biopsied samples in solid tumors.

523 The other relevant issue approached in this study is the discrimination of point mutations.

524 In large hospitals, several methods are applied for determining the presence of a 525 mutation in certain locus, without identifying the specific variant. Others perform a 526 reaction per each mutation. The consequences are a lack of information limiting the 527 possibilities of modern oncology or an increase of laboratory efforts, reducing the 528 sustainability of the health system. Chip-based detection approaches, such as we have 529 introduced, show the advantage of multiplexing. All investigated variants can be 530 implemented on the same microarray. In addition, the inclusion of controls guarantees 531 the assay reliability. Indeed, the optimized protocol is faster (performed in less than 2.5 $532 \mathrm{~h}$ ) and easier to handle than sequencing.

533 According to the amplitude of described applications based on blocked PCR, the 534 expectations of the blocked RPA are high. Furthermore, the method can potentially 535 support the detection/discrimination of mutations in more health scenarios (e.g. small 536 hospital or clinics). Demonstrated for PIK3CA mutational analysis, the next experimental 537 activities are addressed to expand to other mutations. Then, a better stratification of 538 patients, or division of patients into subgroups based on the molecular characteristics, 539 can be achieved and a subsequent individualized treatment can be assigned.

540

541 Conflict of Interest Statement

542 The authors declare that the research was conducted in the absence of any commercial 543 or financial relationships that could be construed as a potential conflict of interest.

\section{Acknowledgments}

545 PROJECT ONCOMARKER (MINECO RTC-2015-3625-1), CTQ 2013-45875-R, FEDER 546 and GVA PROMETEO 2014/40 


\section{REFERENCES}

549 [1] M. Pajic, C.J. Scarlett, D.K. Chang, R.L. Sutherland, A.V. Biankin, Preclinical 550 strategies to define predictive biomarkers for therapeutically relevant cancer subtypes, 551 Hum. Genet. 130 (2011) 93-101.

552 [2] A. Ziegler, A. Koch, K. Krockenberger, A. Großhennig, (2012). Personalized 553 medicine using DNA biomarkers: a review, Hum. Genet. 131 (2012) 1627-1638.

554 [3] R.E. Shackelford, N.A. Whitling, P. McNab, S. Japa, D. Coppola, D. KRAS 555 Testing a Tool for the Implementation of Personalized Medicine, Gene Cancer, 3 (2012) $556 \quad 459-466$.

557 [4] C.A. Milbury, J. Li, G.M. Makrigiorgos, PCR-based methods for the enrichment 558 of minority alleles and mutations, Clin. Chem. 55 (2009) 632-640.

559 [5] R.E. Board, N.J. Thelwell, P.F. Ravetto, S. Little, M. Ranson, C. Dive, A. Hughes, 560 D. Whitcombe, Multiplexed assays for detection of mutations in PIK3CA, Clin. Chem. 54 561 (2008), 757-760.

562 [6] J. Morlan, J. Baker, D. Sinicropi, Mutation detection by real-time PCR: a simple, 563 robust and highly selective method, PLoS One, 4 (2009), e4584.

564 [7] J.D. Luo, E.C. Chan, C.L. Shih, T.L. Chen, Y. Liang, T.L. Hwang, C.C. Chiou, 565 Detection of rare mutant K-ras DNA in a single-tube reaction using peptide nucleic acid 566 as both PCR clamp and sensor probe, Nucleic Acids Res. 34 (2006) e12-e12.

567 [8] H. Wang, J. Jiang, B. Mostert, A. Sieuwerts, J.W. Martens, S. Sleijfer, J.A. 568 Foekens, Y. Wang, Allele-specific, non-extendable primer blocker PCR (AS-NEPB-PCR) 569 for DNA mutation detection in cancer, J. Mol. Diagn. 15 (2013) 62-69.

570 [9] Y. Jia, J.A. Sanchez, L.J. Wangh,. Kinetic hairpin oligonucleotide blockers for 571 selective amplification of rare mutations, Sci. Rep. 4 (2014) 5921.

572 [10] I. Mancini, C. Santucci, R. Sestini, L. Simi, N. Pratesi, F. Cianchi, R. Valanzano, 573 P. Pinzani, C. Orlando, The use of COLD-PCR and high-resolution melting analysis 574 improves the limit of detection of KRAS and BRAF mutations in colorectal cancer, J. Mol. 575 Diagn. 12 (2010) 705-711.

576 [11] Y. Samuels, T. Waldman, Oncogenic mutations of PIK3CA in human cancers, 577 Curr. Top Microbiol Immunol. 347 (2010) 21-41

578 [12] G. Cathomas, PIK3CA in colorectal cancer, Front. Onco., 4 (2014) 35

579 [13] K.D. Courtney, R.B. Corcoran, J.A. Engelman, The PI3K pathway as drug target 580 in human cancer, J. Clin. Oncol. 28 (2010) 1075-1083.

581 [14] C. O'Brien, J.J. Wallin, D. Sampath, D. GuhaThakurta, H. Savage, E.A. 582 Punnoose, P. Guan, L. Berry, W.W. Prior, L.C. Amler, M. Belvin, L. Friedman, M. 
583 Lackner, Predictive biomarkers of sensitivity to the phosphatidylinositol 3' kinase inhibitor

584 GDC-0941 in breast cancer preclinical models, Clin. Cancer Res. 16 (2010) 3670-3683.

585 [15] Y. Zhao, F. Chen, Q. Li, L. Wang, C. Fan, Isothermal amplification of nucleic 586 acids, Chem. Rev, 115 (2015) 12491-12545.

587 [16] C.D. Ahrberg, A. Manz, B.G. Chung, Polymerase chain reaction in microfluidic 588 devices, Lab Chip, 16 (2016), 3866-3884.

589 [17] S. Ikeda, K. Takabe, M. Inagaki, N. Funakoshi, K. Suzuki, Detection of gene point 590 mutation in paraffin sections using in situ loop-mediated isothermal amplification, Pathol. 591 Int. 57 (2007) 594-599.

592 [18] Y.S. Jiang, S. Bhadra, B. Li, Y.R. Wu, J.N. Milligan, A.D. Ellington, Robust strand 593 exchange reactions for the sequence-specific, real-time detection of nucleic acid 594 amplicons, Anal. Chem. 87 (2015) 3314-3320.

595 [19] M. Itonaga, I. Matsuzaki, K. Warigaya, T. Tamura, Y. Shimizu, M. Fujimoto, F. 596 Kojima, M. Ichinose, S.I. Murata, Novel Methodology for Rapid Detection of KRAS 597 Mutation Using PNA-LNA Mediated Loop-Mediated Isothermal Amplification, PloS one, $598 \quad 11(2016)$ e 0151654.

599 [20] Q. Su, D. Xing, X. Zhou, Magnetic beads based rolling circle amplification600 electrochemiluminescence assay for highly sensitive detection of point mutation, 601 Biosens. Bioelectron. 25 (2010) 1615-1621.

602 [21] E.S. Yamanaka, L.A. Tortajada-Genaro, A. Maquieira, Low-cost genotyping 603 method based on allele-specific recombinase polymerase amplification and colorimetric 604 microarray detection, Microchim. Acta, 184 (2017) 1453-1462.

605 [22] S. Galbiati, F. Damin, P. Pinzani, I. Mancini, S. Vinci, M. Chiari, C. Orlando, L. 606 Cremonesi, M. Ferrari, A new microarray substrate for ultra-sensitive genotyping of 607 KRAS and BRAF gene variants in colorectal cancer, PLoS one, 8 (2013) e59939.

608 [23] L.A. Tortajada-Genaro, R. Puchades, A. Maquieira, Primer design for SNP 609 genotyping based on allele-specific amplification-Application to organ transplantation 610 pharmacogenomics, J. Pharm. Biomed. Anal. 136 (2017) 14-21.

611 [24] O. Piepenburg, C.H. Williams, D.L. Stemple, N.A. Armes, DNA detection using 612 recombination proteins, PLoS Biol. 4 (2006) e204.

613 [25] Santiago-Felipe, S., Tortajada-Genaro, L. A., Morais, S., Puchades, R., \& 614 Maquieira, Á. Isothermal DNA amplification strategies for duplex microorganism 615 detection. Food Chem. 174 (2015) 509-515.

616 [26] Y. Liu, T. Lei, Z. Liu, Y. Kuang, J. Lyu, Q. Wang, A Novel Technique to Detect 617 EGFR Mutations in Lung Cancer, Int. J. Mol. Sci. 17 (2016) 792. 
618 [27] S. Santiago-Felipe, L.A. Tortajada-Genaro, S. Morais, R. Puchades, A. 619 Maquieira, One-pot isothermal DNA amplification-Hybridisation and detection by a disc620 based method, Sens. Actuators B Chem. 204 (2014) 273-281.

621 [28] A. Bhattacharyya, C.M. Klapperich, Mechanical and chemical analysis of plasma 622 and ultraviolet-ozone surface treatments for thermal bonding of polymeric microfluidic 623 devices, Lab Chip, 7 (2007) 876-882.

624 [29] Y. Li, Z. Wang, L.M. Ou, H.Z. Yu, DNA detection on plastic: surface activation 625 protocol to convert polycarbonate substrates to biochip platforms, Anal. Chem. 79 (2007) 626 426-433.

627 [30] R. Patel, A. Tsan, R. Tam, R. Desai, J. Spoerke, N. Schoenbrunner, T.W. Myers, 628 K. Bauer, E. Smith, R. Raja, Correction: Mutation Scanning Using MUT-MAP, a High629 Throughput, Microfluidic Chip-Based, Multi-Analyte Panel, PLoS one, 8 (2013) 10-1371. 630 
632 Figure 1. Schematic illustration of the RPA reaction for minority allele enrichment based 633 on the addition of blocking agent. (Up) Wild-type blocked amplification. (Bottom) Mutant 634 amplification. Initial number of copies: $N_{0}$ for wild-type and $N_{0}^{\prime}$ for mutant.

635 Figure 2: (a) Signal registered depending on the oligonucleotide added to a RPA mixture 636 without upstream primer: unmodified blocker (without modification in 3'-end), ddC637 blocker: blocker with 2',3'-dideoxycytidine in 3'-end, tail-blocker: blocker with a 3-mer tail 638 in 3'-end, and C-: negative control oligonucleotide. (b) Signal registered depending on 639 amplification mixture (PCR and RPA) in conventional, blocked with a non-overlapped 640 oligonucleotide and blocked with an overlapping oligonucleotide (blocker concentration $641200 \mathrm{nM})$. T-test: * $p$-value $<0.05,{ }^{* *} p$-value $<0.01,{ }^{* * *} p$-value $<0.001$. Wild-type genomic 642 DNA: 1300 copies.

643 Figure 3: Effect of blocking concentration on the end-point response of RPA-based 644 methods: reactions for exon 9 (a) and exon 20 (b) for wild-type and mutant samples. 645 Discontinue lines correspond to the regression curve applying four-parameter logistic 646 model.

647 Figure 4: Probe selectivity evaluated as the spot intensity of array probes obtained from 648 different unblocked RPA reactions and samples. Dashed line indicates signal of negative 649 control.

650 Figure 5: On-chip hybridization images for oncological patient samples: (a) Microarray 651 layout, (b) Blocked RPA for exon 9, sample: wild-type, (c) Blocked RPA for exon 9, 652 sample: mutant p.E545K, (d) Blocked RPA for exon 20, sample: wild-type, and (e) 653 Blocked RPA for exon 20, sample: mutant p.H1074R.

654

655 LIST OF TABLES

Table 1: (a) Design criteria of blocking oligonucleotide. (b) Optimized variables for 657 blocked RPA.

658 Table 2: Regression parameters obtained applying four-parameter logistic model to 659 experimental data: blocking concentration vs. end-point response of RPA-based 660 methods.

661

662 


\section{SUPPLEMENTARY MATERIAL}

664 Table SI.1: Summary about mutational information of PIK3CA gene: exon 9 and 20.

665 Table SI.2: List of tested oligonucleotides.

666 Table SI.3: Estimated free energy variation $\left(\mathrm{kcal} \mathrm{mol}^{-1}\right)$ for the formation of DNA

667 complexes between probes and templates. The bold numbers correspond to perfect-

668 match complexes.

669

670 Figure SI.1: Images of agarose gel electrophoresis after amplifying PIK3CA gene exon

671 20: (a) PCR products and (b) RPA products after centrifugation-based purification. 1:

672 Ladder, 2: Without blocking agent, 3: Blocker at $50 \mathrm{nM}, 4$ : Blocker at $300 \mathrm{nM}$. Sample:

673 Wild-type template (human cell line).

674 Figure SI.3: Identification of mutation based on chip hybridization assay: (a) Surface 675 activation (photochemical treatment), (b) immobilization of probes (amide bond 676 formation), (c) incubation with RPA products, (d) image developing.

677 Figure SI.4: Spot intensity of array probe for mutation p.H1047R obtained from different 678 concentrations of mutant DNA. Target region: exon 20. Sample: cell culture HCT 116. 679 Linear model: $y=(94 \pm 3) x+(10 \pm 160), R^{2}=0.994$. Limit of detection: $5.1 \%$.

680 
$682 \quad$ (a)

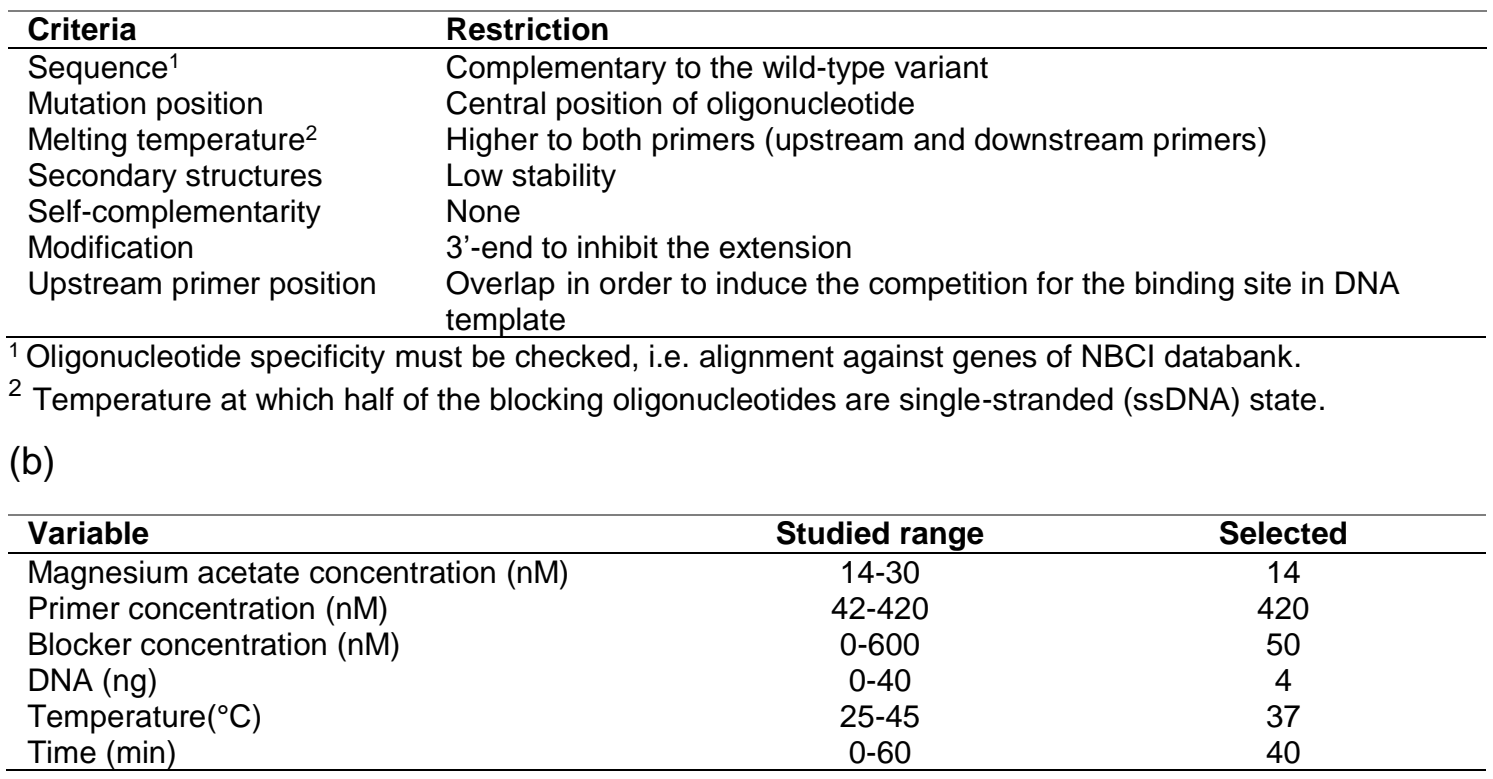

686

$683{ }^{1}$ Oligonucleotide specificity must be checked, i.e. alignment against genes of NBCl databank.

$684{ }^{2}$ Temperature at which half of the blocking oligonucleotides are single-stranded (ssDNA) state.

$685 \quad$ (b)

687 
TABLE 2

\begin{tabular}{lllcccc}
\hline & & a & b & c & d & $\mathbf{R}^{2}$ \\
\hline exon 9 & wild-type & $10000 \pm 300$ & $1.5 \pm 0.3$ & $62 \pm 8$ & $700 \pm 300$ & 0.990 \\
& mutant p.E545K & $10000 \pm 200$ & $1.5 \pm 0.2$ & $140 \pm 14$ & $600 \pm 400$ & 0.995 \\
exon 20 & wild-type & $10500 \pm 400$ & $1.5 \pm 0.2$ & $22 \pm 2$ & $800 \pm 400$ & 0.980 \\
& mutant p.H1047R & $10000 \pm 300$ & $1.5 \pm 0.2$ & $44 \pm 5$ & $800 \pm 300$ & 0.982 \\
\hline
\end{tabular}

689

690 